\title{
Application of image processing to the vehicle license plate recognition
}

\author{
Chen Chunyu \\ Department of Physical and Electricity Information \\ Engineering \\ Daqing Normal University \\ Daqing, 163712, China \\ E-mail:ccy08190309@163.com \\ Cheng Baozhi, Chen Xin \\ Department of Physical and Electricity Information \\ Engineering \\ Daqing Normal University \\ Daqing, 163712, China
}

\author{
Wang Fucheng \\ College of Engineering \\ Heilongjiang Bayi Agricultural University \\ Daqing, 163319, China \\ E-mail:fuchengwang@163.com
}

\author{
Zhang Chen \\ College of Electronic Science \\ Northeast Petroleum University \\ Daqing, 163712, China
}

\begin{abstract}
At present, the traffic engineering and automation have developed, and the vehicle license plate recognition technology need get a corresponding improvement also. In case of identifying a car license picture, the principle of automatic license plate recognition is illustrated in this paper, and the processing is described in detail which includes the preprocessing, the edge extraction, the license plate location, the character segmentation, the character recognition. The program implementing recognition is edited by Matlab. The example result shows that the recognition method is feasible, and it can be put into practice.
\end{abstract}

Keywords-Image processing ; Plate license ; Image extracting; Plate license recognition; Character recognition

\section{INTRODUCTION}

As a vehicle identification, car license plate recognition technology has become a concern topic in automatic control field and in traffic engineering field[1]. It is widely applied to traffic detection, traffic control and guidance, anti-theft security monitoring in public parking, intersection monitoring, vehicle red light violation monitoring, vehicle management in the port and airport access, vehicle management in residential area and other aspects. So it produces a profound effect in social life. The vehicle license plate image processing consists of five modules. They are the pre-processing, the edge extraction, the license plate location, the character segmentation, the character recognition respectively.

The strong image function of Matlab makes it become the first choice of image processing. Each module program is edited by Matlab and they complete license plate recognition eventually.

\section{The Pre-Processing And The Edge EXTraction}

In the transmission process, image is affected by many factors, which leads to some differences with the original image. The image pre-processing is that the useless information such as noise is removed from the acquired image information and the useful information is strengthened by enhancing the boundary effect and increasing the boundary brightness ${ }^{[2]}$. Thus it

is helpful to process the image further. Figure. 1 is an original car photo.

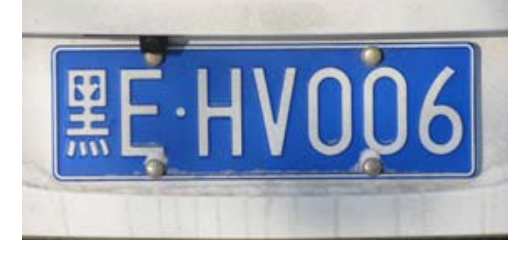

Figure 1. An original car photo

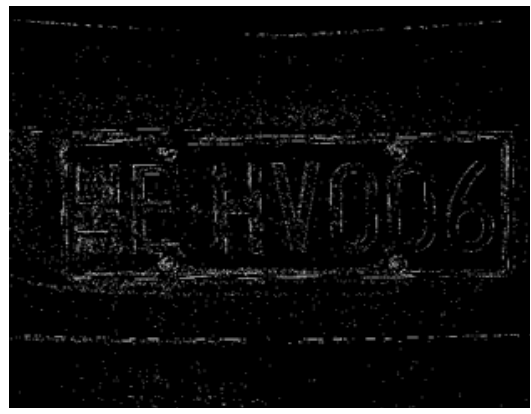

Figure 2. The edge of the image

Figure.2 is the edge of the image which is extracted directly without being filtered. The image edge is prominent and clear, and it contains all the useful information. Thereby Figure. 2 is chosen as the post-processing image of the car identification.

\section{The LiCENSE Plate LOCATION}

After the edge extraction, the original photo of the license plate area turns into the Figure.2. The peak point detection is adopted as the license plate area position method. And the micro-position technology is chosen to deal with the preliminary position further. Shown in Figure.2, the edge 
image $\varphi(x, y)$ is calculated by first order differential operation in the horizontal direction. That is

$$
\begin{aligned}
& \varphi(i, j)=\varphi(i, j)-\varphi(i, j+1) \mid \\
& \text { Among } \quad \text { them, } \quad i=0,1,2,3, \cdots, r-1
\end{aligned}
$$

$j=0,1,2,3, \cdots, l-1, r$ is the row number of the image, and $l$ is the column number of the image. The pixels of image generated by differential operation in the horizontal direction is accumulated in order to get a projection drawing $P(i)$, as is shown in Figure.3.

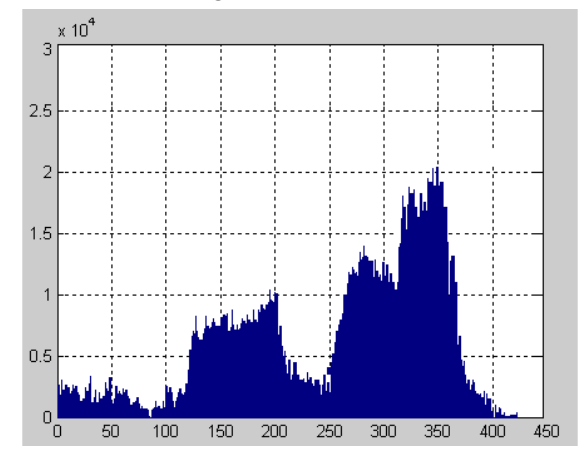

Figure 3. The projection drawing of the edge image in the horizontal direction

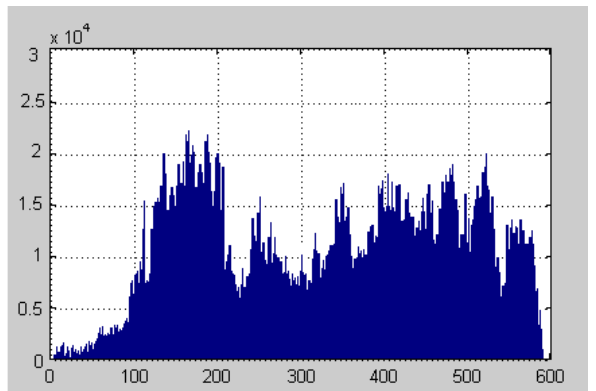

Figure 4. The projection drawing of the edge image in the vertical direction

Generally, the projection value $P(i)$ in the license plate location is larger than other positions, and the projection value $P(i)$ nearby the license plate location plate location is smaller than other positions where exists the wave trough. Finding the two troughs close to the maximum value, the position of the car license plate can be gotten basically. Thus the search scope of the license plate is reduced. As is shown in Figure.3, we can conclude that the license plate location is about between the 310th to the 370th line. Similarly, Figure. 4 in the vertical direction is obtained. So we can infer initially that the license plate location is about between the 110th to the 220th column. The approximate license location is shown in Figure.5.

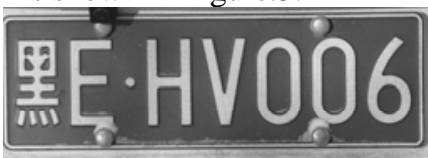

Figure 5. The car license plate positioned initially
The car license plate positioned initially need to be micro-positioned in order to reduce the upper, the lower, the left, and the right boundaries. The specific implementation is as follows:

(1) According to the gray change analysis, the upper and the lower boundaries of the small inclination license plate can be repositioned again. The lateral position of the large inclination license plate requires relevant technical correction.

(2) The car license plate positioned initially need be scanned sequentially from left to right. Once the gray value is greater than the setting value 70 , the scan need be stopped.

\section{The Charcter Segmentation}

After the license plate position is determined, each character of the license plate need to be split, and the resultant image is input to the identification module. Character segmentation in this process plays an important role. The character recognition algorithms are many and the vertical area projection method is often adopted. At the gap between the characters, the projection of the character blocks in the vertical direction must be least in the location, and it meets the writing format and the character size of the license plate $^{[3]}$. After the character segmentation, the image is shown in Figure.6.

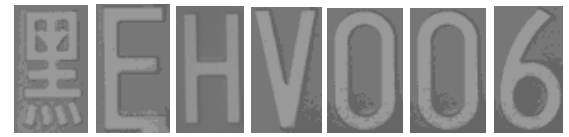

Figure 6. The image after the character segmentation

\section{THE CHARACTER RECOGNITION}

At present, the algorithms of the license plate character recognition mainly have the template matching algorithm and the artificial neural network algorithm ${ }^{[4][5]}$. The template matching algorithm compared with the artificial neural network algorithm is implemented simply, and it has an antiinterference ability, and it has a high recognition accuracy.

Common matching methods have many, such as fourgray template matching, simple binary template matching, projection sequence feature matching, four-cycle area coding look-up table method, the peripheral contour matching method, the strokes direction density characteristics method, etc.

The flow chart of the character recognition algorithm process is as follows:

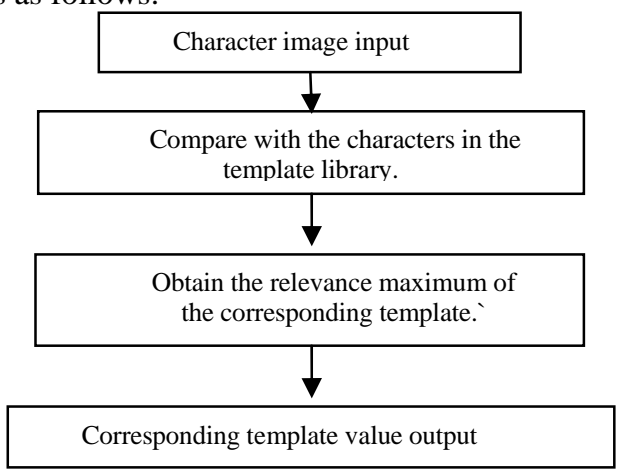


The character recognition is to make a character image normalize, then to convert the normalized image to text format or to display the normalized image in the database directly. Identification consists of two parts. One is Chinese character recognition, the other is non-Chinese character recognition. The key part of the identify is the feature extraction and the classification organization.

Before the character recognition, the first task is to set up a corresponding template library. The number of Chinese characters used in the license plate is about 50. The number of capital letter used in the license plate is 26 . The number of figure used in the license plate is 10 . So the template library is relatively easy to build.

After the character segmentation, the features are extracted, and then they need have a match operation with the characters in the template library. The match degree is determined by the relevant operator ${ }^{[6-8]}$.

Use $S$ the input function to represent the character of the input image and use $T$ the function to represent the template character. Both sizes are $X \times Y$. The match similarity between the unknown and the template is

$$
R=\frac{\sum_{y=1}^{Y} \sum_{x=1}^{X}[S(x, y) \times T(x, y)]}{\sum_{y=1}^{Y} \sum_{x=1}^{X}[S(x, y)]^{2}}
$$

The character recognition is carried out by the program edited by Matlab. The run result is black, E, H,V,0,0 and 6 . The license plate is recognized accurately.

\section{CONCLUSION}

Based on the combination of theoretical research and practice, the image processing to the vehicle license plate is implemented by the program edited by Matlab. And the result is visual. Through the example, we can draw a conclusion that the method can recognize the license plate and the recognition accuracy is high. The method is simple , and can be applied in practice, and has a good application prospect.

\section{ACKNOWLEDGMENT}

1. Youth Foundation of Daqing Normal University research project (Serial number: 09ZQ08)

2. Higher Education Reform project in Heilongjiang Province.

Standy on the Teaching Mode and Practice of Mechanical drawing.(Serial number: JG2012010455)

\section{REFERENCES}

[1] Liu Tong. Research on Vehicle License Plate Recognition System and Related Techniques[D]. Shandong: Shandong university of science and technology, 2005.

[2] Qiu Chengqun. A License Plate Recognition System Based on MATLAB Image Processing [J]. Mechanical Engineer, 2008, (8): 8789.

[3] Niu Xin, Shen Lansun. Research on Vehicle License Plate Recognition Technology[J]. Measurement \& Control Technology, 1999, 18(12): 14-17.

[4] Liu Xinyu. Research on Vehicle License Plate Recognition System Application [D].Zhengzhou: Zhengzhou University, 2004.

[5] Wu Wenqin. Research and Implementation on Car License Plate Recognition Related Techniques [D].Sichuan: Southwest Jiaotong University, 2003.

[6] Xing Xianghua, Gu Guohua. Method of Quickly Recognizing Vehicle Plate Based on Pattern Matching and Characteristic Dot Matching[J].Optoelecfronic Technology, 2003, 23(4): 268-270.

[7] Huang Tao. The application of template matching in the pattern recognition of digital image [J]. Journal of Yunnan University (Natural Sciences), 2005, 27(5): 327-332.

[8] Tang Jin, Li Qing. Fast template matching algorithm[J]. Journal of Computer Applications, 2010, 30(6): 1559-1561. 